\title{
EFECTO DE RECUBRIMIENTOS COMESTIBLES A BASE DE ALMIDÓN NATIVO Y OXIDADO DE YUCA SOBRE LA CALIDAD DE MANGO (Tommy Atkins)
}

\section{EFFECT OF EDIBLE COATINGS BASED ON NATIVE AND OXIDIZED CASSAVA STARCH ON QUALITY OF MANGO (Tommy Atkins)}

\author{
Jorge A. Figueroa1*, Jairo G. Salcedo², Germán J. Narváez ${ }^{3}$
}

Recibido para publicación: Agosto 22 de 2013 - Aceptado para publicación: Diciembre 13 de 2013

\section{RESUMEN}

En relación a las significativas pérdidas poscosecha y la importancia agroindustrial de su cultivo en Colombia, se evaluó el efecto de recubrimientos comestibles a base de almidones modificados de yuca en la conservación del mango (Mangífera índica) variedad Tommy atkins. Para tal fin se formularon películas de almidón nativo y oxidado de yuca (15\%), con glicerolcomo plastificante (10\%) y un compuesto lipídico (3\%). Durante la experiencia se determinó la tasa de respiración, pérdida de peso, acidez titulable, sólidos solubles totales (SST) y $\mathrm{pH}$ del mango, durante 16 días de almacenamiento a temperatura de $20 \pm 2^{\circ} \mathrm{C}$. El uso de recubrimientos logró disminuir significativamente $(\mathrm{p}<0.05)$ el índice de respiración y transpiración de los frutos. El almacenamiento del mango tratado con almidones oxidados de yuca $(A O L)$, resultó ser el mejor tratamiento $(p<0.05)$ en la retención de las propiedades fisicoquímicas evaluadas.

Palabras clave: Almidón, Frutas, Pérdida de peso, Poscosecha, Respiración.

\begin{abstract}
In relation to the significant post-harvest losses and agro-industrial importance of cultivation in Colombia, the effect of edible coatings based on modified cassava conservation Mango (Mangifera indica) variety Tommy Atkins starches was evaluated. For this purpose native and oxidized cassava starch films (15\%) were formulated, with glycerol as a plasticizer (10\%) and a lipid compound (3\%).During the experiment it was determined the respiration rate, weight loss, acidity, total soluble solids (TSS) and $\mathrm{pH}$ of the handle, for 16 days of storage at $20 \pm 2{ }^{\circ} \mathrm{C}$. The use of coatings was able to significantly decrease $(p<0.05)$ the rate of respiration and transpiration of fruits.

The storage of Mango treated with cassava's oxidized starches $(A O L)$, proved to be the best treatment $(p<0.05)$ in retention of physicochemical properties evaluated.

Key words: Starch, Fruit, Weight Loss, Postharvest, Breathing.

"Ingeniero Agroindustrial. Joven Investigador. Universidad de Sucre - COLCIENCIAS. Cra 28 № 5-267, Sincelejo (Sucre), Teléfono (5) 282 12 40, e-mail: jorgefig12_@hotmail.com

²P.D en Ingeniería Química. Facultad de Ingeniería. Universidad de Sucre. Sincelejo, Colombia.

${ }^{3}$ Ingeniero Agroindustrial. Especialista en Ingeniería de Procesos de Alimentos y Biomateriales. Facultad de Ingeniería. Universidad de Sucre. Sincelejo, Colombia.
\end{abstract}




\section{INTRODUCCIÓN}

El cultivo de mango ostenta un importante eslabón en el contexto agrícola colombiano, ya que se producen aproximadamente 341.964 toneladas. Sin embargo, cerca del $30 \%$ de la producción se cuantifica como pérdidas debido al inadecuado manejo poscosecha (MADR 2010).

El mango, es un fruto climatérico, y tiene unavida en anaquel muy corta. Su comercialización se realiza en un período muy reducido, pues es un producto altamente perecedero y susceptible al deterioro generado por desórdenes fisiológicos y patológicos en la poscosecha. Estos desórdenes son en gran medida los causantes de deshidratación, produciendo disminuciones en el peso, cambios indeseables en la textura y apariencia de los frutos (Cáceres et al. 2003).

Debido al deterioro acelerado que sufre éste producto hortofrutícola, se han estudiado diversas alternativas que ayuden a preservar la calidad global (organoléptica, comercial, microbiológica y nutritiva), disminuir las pérdidas y satisfacer las crecientes exigencias del mercado. En este sentido, el uso de recubrimientos comestibles ha surgido como alternativa para mantener la calidad de los frutos, ya que mejora su apariencia y prolonga su vida comercial y regula sus procesos fisiológicos (Báez et al. 2000).

Con el fin de aprovechar las diversas ventajas que aportan los componentes de naturaleza lipídica e hidrofílica a las formulaciones de recubrimientosy decontrarrestarlas deficiencias que presentan por separado, se han venido utilizando mezclas de estos componentes. Los lípidos aportan la barrera al vapor de agua y los hidrocoloides la permeabilidad selectiva al $\mathrm{CO}_{2}$ y $\mathrm{O}_{2}$. Entre los hidrocoloides estudiados para el desarrollo de películas y recubrimientos comestibles se encuentran: celulosa y sus derivados, metilcelulosa, alginatos, pectinas, goma arábiga, almidones y almidones modificados (Pérez et al. 2008).

En la actualidad, debido a su abundancia y bajo costo de producción, existe un gran interés en la utilización de almidón como componente de películas comestibles y recubrimientos biodegradables (Palacín 2012). Las películas elaboradas a partir de este carbohidrato son claras, flexibles, transparentes y presentan excelentes barreras al oxígeno. Sin embargo, sus propiedades de barrera frente a la humedad, no son tan buenas, y sus propiedades mecánicas son muy inferiores a las películas sintéticas (Rivas et al. 2008).

Las limitaciones anteriores se pueden superar modificando la estructura del almidón nativo por métodos químicos, físicos y enzimáticos dando como resultado un almidón modificado; se incluye a los almidones oxidados, entrecruzados y acetilados. Estos almidones generalmente muestran mejor claridad de pasta y estabilidad, mejora de sus propiedades mecánicas en la formación de películas, disminución de la viscosidad y mejora de la tendencia a la retrogradación (Agboola et al. 1991). No obstante, Zamudio et al. (2007) argumenta que el carácter hidrofílico de estas películas les confiere un aspecto quebradizo causado por las altas fuerzas intermoleculares. Los plastificantes como glicerol, sorbitol, polietilenglicol, aumentan la flexibilidad de las 
películas debido a su capacidad para reducir los enlaces de hidrógeno internos entre las cadenas de los polímeros mientras aumentan el espacio molecular. Los plastificantes más usados en las películas de almidón son el sorbitol y el glicerol (Mali et al. 2005).

Algunos usos de almidones que han sido objeto de investigación como recubrimientos incluyen muestran excelentes propiedades mecánicas y de barrera a los gases incluyendo: (1) almidón de yuca plastificado con glicerol y polietilenglicol (Parra et al. 2004); el anterior incorporado con compuestos antimicrobianos naturales (Kechichian et al. 2010), (2) almidón de maíz estándar y pre-gelatinizado (Pagella et al. 2002); (3) almidón de yuca formulado con ácido ascórbico, $\mathrm{N}$-acetil-cisteína en la calidad del plátano (Palacín 2012):(4) almidón de yuca y proteína de soja en la conservación de fresas (Saavedra y Algecira 2010); (5) almidón modificado de yuca en la maduración del tomate (Hernández et al. 2011); y (6) almidón de yuca y papa en la conservación del mango cv. Zapote (Trujillo et al. 2011).

Según Palacín (2012), el desarrollo de recubrimientos a base de polisacáridos ha conllevado un incremento significativo en las clases de aplicaciones que pueden tener y la variedad de productos que pueden ser tratados, para extender la vida de anaquel de los vegetales.

En consecuencia, en el presente trabajo se busca formular y evaluar recubrimientos comestibles a base de almidones modificados de yuca para determinar el mejor tratamiento en a conservación del mango, a través, de la valoración de propiedades fisicoquímicas y fisiológicas durante el almacenamiento.

\section{MATERIALES Y MÉTODOS}

\section{Material Experimental.}

Durante la investigación se utilizó almidón nativo de yuca variedad M-Tai, procedente de la planta "Almidones de Sucre S.A.S". Las frutas utilizadas para el estudio fueron mangos (Mangífera indica, variedad Tommy atkins), cosechados en el vivero Agrocampos ubicado en municipio de Sincelejo.

Los mangos considerados fisiológicamente maduros (grado 3, según NTC 5210/2003), se seleccionaron libre de magulladuras, golpes y alteraciones microbiológicas. Además que presentarán peso entre 450 a 500 g, y características físicas (tamaño y forma) homogéneas. Luego de la etapa de recolección las frutas se sometieron a un tratamiento previo donde fueron lavadas, desinfectadas (agua clorada a 50.0 ppm), almacenadas y refrigeradas a temperatura de $15 \pm 2^{\circ} \mathrm{C}$.

El proceso de oxidación del almidón se realizó con hipoclorito de sodio $(\mathrm{NaOCl})$ al 3,0\% $\mathrm{p} / \mathrm{v}$, siguiendo la metodología propuesta por Rivas et al. (2008).

Los recubrimientos fueron preparados utilizando una mezcla de almidón nativo y oxidado (15 g/kg), glicerol (10 g/kg) y agua (975 g/kg de solución), basados en estudios previos realizados por Trujillo et al. (2012) y Palacín (2012). Los componentes semezclaron y homogenizaron en un agitador magnético con plataforma de calentamiento (Thermo lyne Nuova II S1842) por 30 minutos controlando la temperatura hasta $70 \pm 2^{\circ} \mathrm{C}$, eludiendo la pregelatinización del almidón. La homogenización continuó por 20 minutos más 
hasta descenso de la temperatura a $35 \pm 2^{\circ} \mathrm{C}$.

Con el objetivo de incrementar el carácter hidrofóbico de algunas de laspelículas utilizadas en el presente experimento, se adicionó 3,0 g/ kg de aceite de ajonjolí a la solución formadora de recubrimiento, utilizando lecitina de soya $(3,0 \mathrm{~g} / \mathrm{kg}$ de solución) como emulsificante. En total se prepararon tres recubrimientos, los cuales se detallan a continuación:

AN: Almidón nativo + agua + glicerol AO: Almidón oxidado + agua + glicerol AOL: Almidón oxidado + agua + glicerol + compuesto lipídico (aceite de ajonjolí)

Para establecer el efecto de las películas elaboradas a partir de almidón de yuca en lavida poscosecha de los mangos, se realizaron pruebas a modo de testigo (B: Blanco). Los recubrimientos se aplicaron mediante la técnica de frotación, utilizando una espuma estéril, hasta alcanzar una capa homogénea en la superficie de los frutos. Finalmente, los productos recubiertos se situaron en bandejas en lugar ventilado durante 5 minutos, para luego ser almacenados a temperatura de $20 \pm 2^{\circ} \mathrm{C}$ y $902 \%$ de $\mathrm{HR}$.

\section{Variación de propiedades fisiológicas}

\section{Tasa de respiración.}

La tasa de respiración se determinó por el método de titulación, basados en la producción de $\mathrm{CO}_{2}$ de las frutas (Angueira et al. 2003). Para tal efecto, se implementó un sistema de captura de $\mathrm{CO}_{2}$ acoplado a una unidad de refrigeración con termostato para el control de la temperatura. Este proceso se realizó por triplicado para cada tratamiento establecido, a temperatura de 20 $2^{\circ} \mathrm{C}$ durante 16 días de almacenamiento, y los resultados se expresaron en $\mathrm{mgCO}_{2} \mathrm{Kg}^{-1} \mathrm{~h}^{-1}$.

\section{Pérdida de peso.}

Se pesaron las muestras al inicio $y$ final de cada día de almacenamiento, durante 16 días a una temperatura de $20 \pm 2^{\circ} \mathrm{C}$, utilizando una balanza analítica Mettler Toledo PR 8002. Los resultados obtenidos se expresaron como porcentaje de pérdida de peso respecto al peso inicial. Se estableció un número de cinco réplicas por tratamiento.

\section{Variación de propiedades fisicoquímicas}

Acidez titulable, $\mathrm{pH}$ y sólidos solubles totales. La acidez titulable se determinó siguiendo el procedimiento $942.15 / 90$ descrito por la AOAC, expresado en porcentaje de ácido cítrico. Utilizando un $\mathrm{pH}$-metro previamente calibrado a temperatura ambiente se indicó el $\mathrm{pH}$ del jugo de fruta, según el método 981.12/90 de la AOAC. La concentración de sólidos solubles totales (SST) se determinó siguiendo el método 932.12/90 de la AOAC, con corrección por acidez (ICONTEC, 1996) por medio de la ecuación:

S.S.T corregidos $=0.194 * A+$ S.S.T; Donde $\mathrm{A}=\%$ de ácido cítrico,

\section{S.S.T = Sólidos solubles totales}

\section{Diseño experimental.}

El experimento se estudió bajo un diseño factorial completamente aleatorizado. Los factores establecidos fueron tipo de recubrimiento en cuatro niveles $(B, A N, A O$, AOJ) y tiempo de almacenamiento en ocho niveles $(2,4,6,8,10,12,14,16$ días). Para la interpretación de resultados se realizó un 
análisis de varianza con probabilidad de error del 5\%. Para la comparación de medias se adoptó la prueba de Tukey, utilizando el software estadístico R- versión 3.1.0.

\section{RESULTADOS Y DISCUSIÓN}

\section{Propiedades fisiológicas.}

Durante el experimento los mangos presentaron un aumento significativo en la pérdida de agua $(p<0.05)$ respecto al factor tiempo, con un incremento constante hasta el final del periodo de almacenamiento (Figura 1A). Los frutos sin recubrir (B) presentaron mayor pérdida de peso, con una disminución significativa $(p<0.05)$ en el porcentaje para mangos recubiertos con almidón nativo (AN) y tratados con almidón oxidado formulado con el compuesto lipídico (AOL). Resultados similares fueron reportados por Pérez et al. (2005) en mangos de la misma variedad recubiertos con cera comercial y por Velasco et al. (2012) en mangos recubiertos con una cera comestible formulada a base de carbohidratos.

Lo anterior indica que los recubrimientos AN y $A O L$ permiten reducir la pérdida de agua en mangos almacenados a temperatura de $20 \pm 2^{\circ} \mathrm{C}$, atribuyéndose a las propiedades de barrera de gases que retarda la difusión del vapor de agua

A

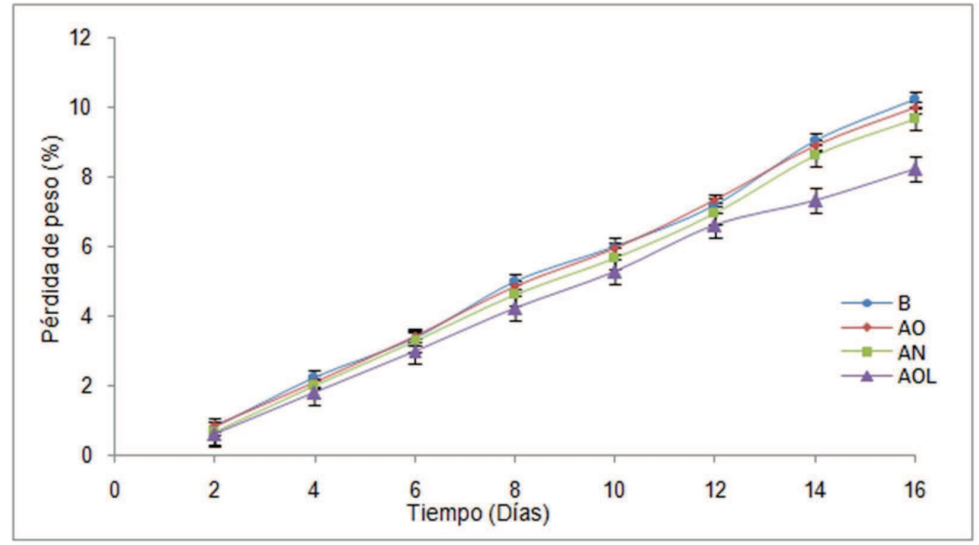

B

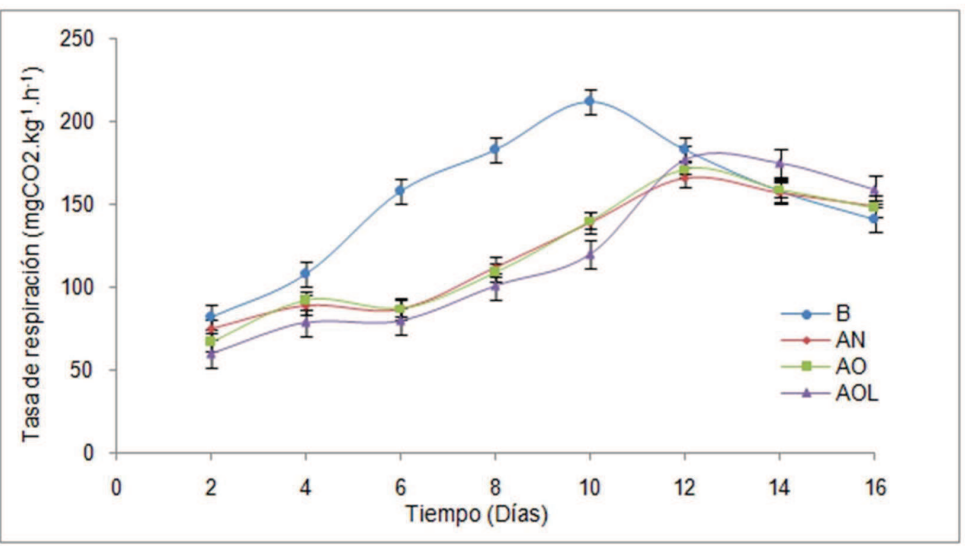

Figura 1. Variación de la pérdida de peso (A) y tasa de respiración (B) en mangos variedad Tommy atkins tratados con recubrimientos y almacenados a $20 \mathrm{C}$ y $90 \% \mathrm{HR}$. 
entre el fruto y el medio exterior. La aplicación de las películas de almidón donde se incorporó el compuesto lipídico evidencia un buen desempeño desde el punto de vista fisiológico sobre los mangos puestas a prueba, esto debido a una mejora en las capacidades de barrera al vapor de agua como resultado del carácter hidrofóbico dado por el aceite. Zamudio et al. (2007a) reporta que películas de almidón oxidado formuladas con aceite de girasol presentaron menor solubilidad, que relaciona con una disminución a la permeabilidad al vapor de agua y capacidad de las películas para reducir la pérdida de humedad en alimentos. Además, algunos investigadores reportan una disminución en la pérdida fisiológica de peso en tomates cubiertos con películas a base de almidón modificado de yuca (Hernández et al. 2011), y una disminución altamente significativa en plátanos utilizando películas a base de almidón nativo de yuca (Palacín 2012).

Sin embargo, no se encontraron diferencias significativas $(p>0.05)$ entre los frutos $\sin$ recubrir (B) y recubiertos con almidón oxidado (AO). Este fenómeno puede ser debido a que los almidones oxidados presentan una mayor permeabilidad al vapor de agua que los almidones nativos, resultado de la formación de grupos carbonilos y carboxilos durante la oxidación del almidón, que le confieren carácter hidrofílicoalaspelículas (Zamudio etal. 2010b).

Al final del período de almacenamiento los frutos no presentaron una adecuada apariencia, asociada a la pérdida de agua, alcanzando pérdidas superiores al 8,0 $\pm 1,12 \%$.Según Souza et al. (2011), la pérdida de peso alrededor del 3 a $6 \%$ son suficientes para causar un marcado descenso en la calidad de los mangos.
Todos los frutos mostraron una producción de $\mathrm{CO}_{2}$ característica de frutos climatéricos, con un aumento significativo $(p<0.05)$ en cuanto al factor tiempo, con la máxima actividad respiratoria entre los 9 y 12 días de almacenamiento (Figura 1B). Los mangos sin recubrir alcanzaron un pico máximo de $\quad 212,02 \pm 7,0 \quad m g \mathrm{mO}_{2} \cdot \mathrm{kg}^{-1} \cdot \mathrm{h}^{-1}$ (aprox. $117,88 \pm 3,8 \mathrm{mLCO} \cdot \mathrm{kg}^{-1} \cdot \mathrm{h}^{-1}$ ) para el día 9 de almacenamiento, tiempo ligeramente superior al reportado por Morais et al. (2003) en mangos Tommy atkins, mientras que se indican resultados similares a los obtenidos alcanzando un máximo de actividad respiratoria en mangos Tommy (Velasco et al. 2012) y en mangos "Ataulfo" a partir del día 8 de almacenamiento (Rangel et al. 2009).

Los frutos recubiertos prolongaron el tiempo del pico climatérico en aproximadamente dos días, asimismo presentaron una disminución significativa $(p<0.05)$ en la actividad respiratoria durante el almacenamiento en comparación con los testigos. Hoa et al. (2002), reportan una reducción similar en la producción de $\mathrm{CO}_{2}$ en mangos cv. Kent y Tommy atkins tratados con diferentes recubrimientos. Esto indica que los recubrimientos a base de almidones modificados de yuca aplicados en mangos cv. Tommy atkins, retrasan la velocidad de respiración, debido al control que ejercen en el intercambio gaseoso entre éstos y el medio, prolongando su tiempo de vida útil.

La máxima actividad respiratoria se obtuvo en mangos tratados con una película comestible a base de quitosano. Asimismo, el aumento en el tiempo para alcanzar el pico climatérico en mangos recubiertos, se puede relacionar con el efecto que causan laspelículas abase de almidón 
para ralentizar la producción de $\mathrm{CO}_{2}$ y por ende, retrasar la actividad metabólica de los frutos y su velocidad de maduración(Hoa et al. 2002).

Los recubrimientos formulados con almidón nativo (AN) y almidón oxidado ( $\mathrm{AO}$ ) presentan un comportamiento similar en la reducción de la tasa de respiración de los frutos estudiados ( $p>0.05)$. Asimismo, se observa que el tratamiento elaborado con almidón oxidado y el compuesto lipídico (AOL), mostro ser el más efectivo en la disminución de la actividad respiratoria de los frutos respecto a los testigo (B). Chiumarelli y Hubinger (2012), resaltan que los recubrimientos de almidón de yuca formulados con glicerol y cera carnauba (compuesto lipídico) mejoraron las propiedades de barreras de gases del revestimiento, permitiendo disminuir la tasa de respiración en manzanas mínimamente procesadas.

\section{Propiedades fisicoquímicas.}

En la Figura 2, se muestra la variación de la acidez titulable, $\mathrm{pH}$ y sólidos solubles totales (SST) de los frutos tratados en función del tiempo de almacenamiento. La acidez titulable en los mangos decreció significativamente $(p<0.05)$ a los 16 días de almacenamiento, siendo más altas en los frutos tratados comparados con los frutos testigos. Zambrano et al. (2011) utilizando recubrimientos de almidón, metilcelulosa y quitosán reportaron resultados similares.

La acidez titulable en los frutos recubiertos descendióal finaldel periododealmacenamiento a valores promedios de 0,638 $\pm 0,04$ (AN), $0.594 \pm 0.03 \quad(\mathrm{AO})$ y $0,764 \pm 0.04$ (AOL), claramente superior a la alcanzada en frutos

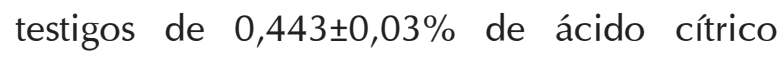
(Figura 2A). Rhatore et al. (2009), asocian este comportamiento a la atmósfera modificada generada por los recubrimientos que permite ralentizar el proceso metabólico en los frutos, y en consecuencia, se retrasa la degradación enzimática del ácido cítrico o conversión del mismo en azúcar durante la maduración.

Estos resultados demuestran un retraso en la maduración de los frutos tratados a temperatura de $20 \pm 2^{\circ} \mathrm{C}$, manifestándose de forma evidente en los frutos recubiertos con almidón oxidado formulado con el compuesto lipídico. Retención de la acidez y extensión del tiempo de vida útil en mangos recubiertos, igualmente fueron reportados por Souza et al. (2003).

En los valores de $\mathrm{pH}$ hubo un incremento significativo $\quad(p<0.05), \quad$ durante el almacenamiento (Figura 2B), este incremento está asociado con el proceso de maduración donde los frutos empiezan a consumir parte de sus ácidos orgánicos como parte de sus procesos metabólicos (Rhatore et al. 2009). Inicialmente los frutos presentaron una media de $3,41 \pm 0,14$ en el valor del $\mathrm{pH}$, el cual

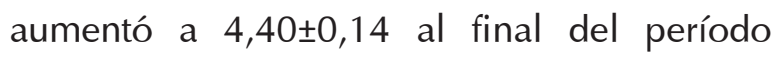
de almacenamiento. Este rango es similar al mostrado por mangos cv. Tommy atkins (Carrera et al. 2009) y en mangos de la misma variedad tratados con una cera comestible a base de carbohidratos (Pérez et al. 2005).

Con la implementación de los recubrimientos a base de almidón nativo (AN) y modificado (AO), los frutos presentaron un leve aumento en el valor de $\mathrm{pH}$ hasta el final del período de almacenamiento, contrario, al comportamiento mostrado por los mangos sin recubrir donde la variación en el valor de $\mathrm{pH}$ fue más evidente de $3,48 \pm 0,17$ hasta $4,77 \pm 0,17$. Resultados 
análogos fueron publicados por Hoa et al. (2002) y Trujillo (2012) en mangos recubiertos a base de almidón almacenados a $20^{\circ} \mathrm{C}$ y $60 \% \mathrm{HR}$.

Las películas formuladas con almidón oxidado y el compuesto lipídico (AOL) mostraron ser la más efectiva, en cuanto a la retención en el cambio del $\mathrm{pH}$ en los frutos. Este comportamiento puede ser causado en la inhibición de la actividad metabólica del fruto relacionada por el efecto positivo del recubrimientoen la extensión del proceso de maduración. Lo anterior, justifica lo señalado por Zamudio et al. (2007a) y Yan et al. (2012), que recomiendan el uso de películas elaboradas a base de almidón oxidado en la conservación de productos hortofrutícolas, debido, a que las películas presentaron excelentes propiedades mecánicas y de barrera de gases. El contenido de solidos solubles totales (SST) en frutos de mango presentó una evolución progresiva durante el almacenamiento, desde

A

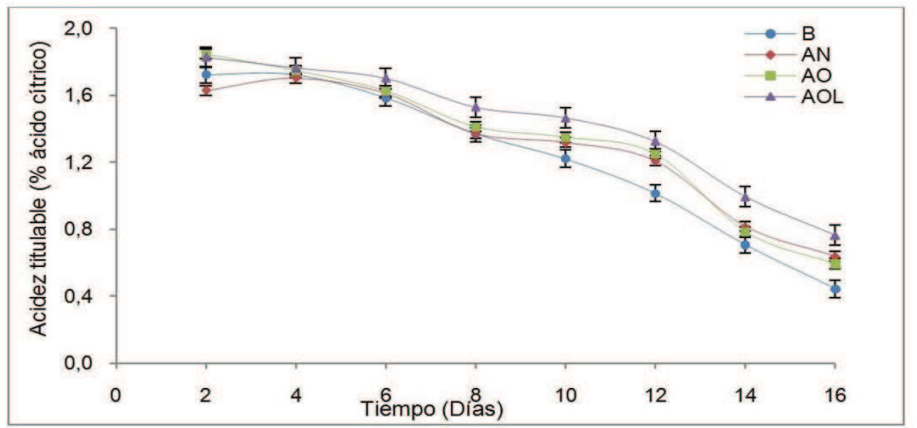

B

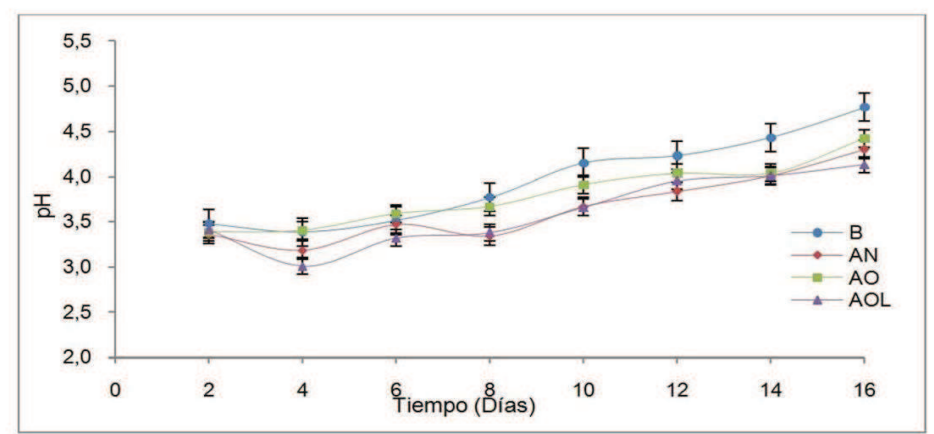

C

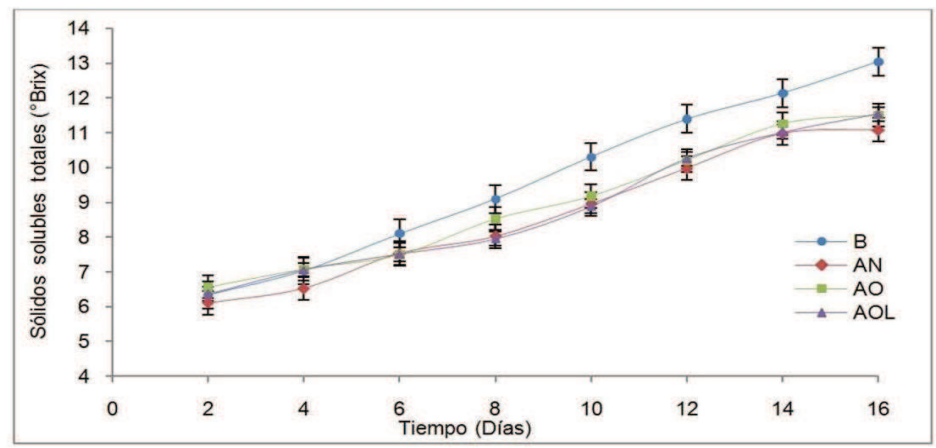

Figura 2. Cambios en acidez titulable (A), $\mathrm{pH}(\mathrm{B})$ y sólidos solubles totales (C) en frutos de mango en mangos variedadTommy atkins tratados con recubrimientos y almacenados $20 \mathrm{C}$ y $90 \% \mathrm{HR}$. 
$6,34 \pm 0,80^{\circ}$ Brix hasta un valor promedio de $11,80 \pm 0,80^{\circ}$ Brix (Figura 2C). Rathore et al. (2009) concluyeron que el incremento de SST puede ser causado por la conversión del almidón presente en el fruto, como resultado de los cambios fisiológicos durante el almacenamiento. Siller et al. (2009) y Osuna et al. (2012) reportaron un aumentó del contenido de SST en mangos cercanos al rango anteriormente mencionado.

Al inicio del período de almacenamiento no hubodiferenciassignificativasentretratamientos ( $p>0.05$ ), en la variación de los sólidos solubles totales (SST) en frutos de mango cv. Tommy Atkins. Sin embargo, el efecto principal de la aplicación de recubrimientos en la variación de los SST es significativo $(p<0.05)$ a partir del octavo día de almacenamiento. Al final del experimento los frutos a los que se aplicaron las películas de $\mathrm{AN}, \mathrm{AO}$ y $\mathrm{AOL}$ alcanzaron $11,09 \pm 0,68,11,51 \pm 0,67$ y $11,54 \pm 0,68^{\circ}$ Brix, respectivamente, contra $13,05 \pm 0,86^{\circ} \mathrm{Brix}$ en el testigo (B). Comportamientos similares fueron registrados por Trujillo et al. (2012) en mango variedad zapote y por Rangel et al. (2009) en mango variedad ataulfo recubiertos con almidón de papa y quitosano, respectivamente.

\section{CONCLUSIONES}

Los resultados del presente estudio sugieren el uso de recubrimientos comestibles en frutos de mango cv. Tommy atkins, por su efectividad en ralentizar el proceso fisiológico de respiración, extendiendo el pico climatérico de estos frutos en dos días adicionales. Mediante el uso de almidón de yuca, se ha conseguido retrasar los cambios en la pérdida de peso y características fisicoquímicas relacionados con la maduración, y por tanto, prolongando la vida útil de los productos.

El análisis estadístico muestra diferencias del control con los tratamientos aplicados, considerando al tratamiento formulado con almidón oxidado y el compuesto lipídico ser el más efectivo como técnica de conservación. Este estudio ha resaltado la excelente la capacidad de formación de película del almidón oxidado, su resistencia al agua, flexibilidad mecánica y excelente barrera al oxígeno en las condiciones de almacenamiento establecidas. Asimismo, la incorporación de productos naturales de diversas fuentes en la formulación de recubrimientos comestibles, se convierte en una interesante alternativa que permite reducir el impacto que tienen sobre el ambiente las prácticas comúnmente aplicadas.

\section{AGRADECIMIENTOS}

Se agradece a la Universidad de Sucre por la financiación de esta investigación, a través, de convocatoria interna reglamentada en la resolución 31 de 2008. Asimismo, se agradece a COLCIENCIAS y el Programa Jóvenes Investigadores e Innovadores "Virginia Gutiérrez de Pineda", por la beca-pasantía otorgada a uno de los autores.

\section{REFERENCIAS}

Agboola, S., Akingbala, O., and Oguntimein, G. 1991. Physicochemical and functional properties of low DS cassava starch acetates and citrates. Starke 43(2): 62-66.

Angueira, M., Sandoval, A., y Barreiro, J. 2003.Tasas de respiración en cuatro híbridos de pimentón (Capsicum Annum L.). Revista 
Interciencia 28(10): 593-596.

AOAC (Association of Oficial Analytical Chemists). 1990. Official methods of analysis, Virginia, p 110, 234, 428.

Báez, R., Bringas, E., Mendoza, A.M., González A.G, y Ojeda A.J. 2000. Recubrimientos de tratamientos especiales en frutos de mango tratados hidrotérmicamente. Villamizar, F.; Báez, R. (eds), Segundo Congreso Iberoamericano de Tecnología Poscosecha. Universidad Nacional de Colombia. Departamento de Ingeniería Agrícola. Bogotá (Colombia), p71-74.

Cáceres, I., Mulkay, T., Rodríguez, J., Paumier, A. y Sisino, A. 2003. Influencia del encerado y tratamiento térmico en la calidad poscosecha del mango. Revista Simiente 73(1): $25-29$

Carrera, A., Gil, R. y Mark, D. 2009. Comportamiento poscosecha de cinco cultivares de mango tratados con $\mathrm{CO} 2$ y almacenados bajo condiciones naturales, en la Estación Experimental de INIA Caripe, estado Monagas. Revista UDO Agrícola 9(1): 51-59.

Chiumarelli, M., and Hubinger, M. 2012. Stability, solubility, mechanical and barrier properties of cassava starch - Carnauba wax edible coatings to preserve fresh-cut apples. FoodHydrocolloids 28(1):59-67.

Hernández, P., Burbano, A., Mosquera, S., Villada, H., y Navia, D. 2011. Efecto del recubrimiento a base de almidón de yuca modificado sobre la maduración del tomate. Revista Lasallista de Investigación 8(2): 96-103.
Hoa, T., Ducamf, M., Lebrup, M. and Baldwin, E. 2002.Effect of different coating treatments on the quality of mango fruit. Journal of FoodQuality 25 (6): 471-486.

ICONTEC (Instituto Colombiano de Normas Técnicas y Certificación). 1996. Productos alimenticios: Frutas, legumbres y hortalizas procesadas. Néctares de frutas. NTC 659, Bogotá, p56.

ICONTEC (Instituto Colombiano de Normas Técnicas y Certificación). 2003. Frutas frescas. Mango. Variedades Mejoradas. Especificaciones. NTC 5210, Bogotá, p32.

Kechichian, V., Ditchfield C., Veiga, P. and Tadini, C. 2010. Natural antimicrobial ingredients incorporated in biodegradable films based on cassava starch. LWT - Food Science and Technology 43(7): 1088-1094.

Mali, S., Sakanaka, L., Yamashita, F., and Grossmann, M. 2005.Water sorption and mechanical properties of cassava starch films and their relation to plasticizing effect. CarbohydratePolymers 60(3): 283-289.

Ministerio de Agricultura y Desarrollo Rural (MADR). 2010. Plan Frutícola Nacional. 2010. Bogotá (Colombia), p145.

Morais, P., Filgueras, H., Pinho, J., Alves, R. and Assis, J. 2003. Vida útil de mangos cv. Tommy atkins recolectados en el estadio de maduración comercial. Revista Iberoamericana de Tecnología Poscosecha 5(1): 26-32.

Osuna, T., Sañudo, J., Rangel, M., Basilio, J., Valdez, B., Hernández, S. y Villareal, M.2012. Aplicación precosecha de etefón 
para mejorar la calidad de mangos 'Tommy atkins' para procesado industrial. Revista Fitotecnia Mexicana 35(5): 69-74.

Pagella, C., Spigno, G. y De Faveri, D. 2002. Characterization of starch based edible coatings. Food and BioproductsProcessing80(3): 193 - 198.

Palacín, J. 2012. Efectos de recubrimientos de almidón de yuca, ácido ascórbico, $\mathrm{N}$-acetil-cisteína en la calidad del plátano (Musa paradisiaca). Tesis de Maestría, Ciencia y Tecnología de Alimentos. Universidad Nacional de Colombia. Cartagena.

Parra, D., Tadini, C., Ponce P. y Lugão, A. 2004. Mechanical properties and water vapor transmission in some blends of cassava starch edible films. Carbohydratepolymers 58: 475 481.

Pérez, B., Bringas, E., Cruz, L. y Báez, R. 2005. Evaluación de cera comestible en mango "Tommy Atkins" destinado a la comercialización para el turismo. Parte I: Efecto en las características físico-químicas. Revista Iberoamericana de Tecnología Poscosecha $7(1): 24-32$.

Pérez, M., Del Rio, M. y Rojas, C. 2008. Recubrimientos comestibles en frutas $y$ hortalizas. Centro de Poscosecha del Instituto Valenciano de Investigaciones Agrarias (IVIA). Revista Horticultura 207: 54-57.

Rangel, D., Espinoza, B., Jorge, C., Sañudo, A., Valdez, B. y Osuna, T. 2009. Efecto del 1-metilciclopropeno (1-MCP) y de una película comestible sobre la actividad enzimática y calidad poscosecha del mango "Ataulfo". Revista Fitotecnia 32(1): 53-60.

Rivas, M., Méndez, M., Sánchez, M., Núñez, M. y Bello, L. 2008. Caracterización morfológica, molecular y fisicoquímica del almidón de plátano oxidado y lintnerizado. Agrociencia 42(5): 487-497.

Saavedra, N. y Algecira N. 2010. Evaluación de películas comestibles de almidón de yuca y proteína de soya en la conservación de fresas. Revista Nova 8(14): 171-182.

Siller, J., Rangel., M., Báez, M, Araiza, E. e Ireta, A. 2009. Calidad poscosecha de cultivares de mango de maduración temprana, intermedia y tardía. Revista Fitotecnia Mexicana 32(1): 45-52.

Souza, M., Ascari, C., Magalhães, K., Mattiuz, C. y Mattiuz, B. 2011. Pós-colheita de mangas 'Tommy atkins' recobertas com quitosana. Revista Brasileira de Fruticultura, Volume Especial: 337-343.

Trujillo, Y., Pérez, J. y Durán, D. 2012. Empleo de recubrimientos comestibles con base en almidón de papa y yuca en la conservación del mango cv. Zapote. Revista Alimentech 10 (1):5-17.

Velasco, B., Mercado, J., García, J. y Báez, R. 2012. Respuesta física y fisiológica a la aplicación de cera comestible en mangos (Mangifera indica)cvs. Tommy atkins y Keitt. Revista Iberoamericana de Tecnología Poscosecha 13(2): 130-139. 
Yan, Q., Hou, H., Guo, P. and Dong, H. 2012.Effects of extrusion and glycerol content on properties of oxidized and acetylated corn starch-based films. CarbohydratePolymers 87(1): 707-712.

Zamudio, P., Bello, L., Vargas, A., Hernández, J. y Romero, C. 2007a. Caracterización parcial de películas preparadas con almidón oxidado de plátano. Revista Agrociencia 41(8): 837-844.
Zamudio, P., Vargas, A., Gutiérrez, F. y Bello, L. 2010b. Caracterización fisicoquímica de almidones doblemente modificados de plátano. Revista Agrociencia 44(3): 283-295. 